Research Paper

\title{
Adjuvant Radiotherapy of Involved Field versus Elective Lymph Node in Patients with Operable Esophageal Squamous Cell Cancer: A Single Institution Prospective Randomized Controlled Study
}

\author{
Ruifeng Liu ${ }^{1,2,3^{*}}$, Xueliang Zhang ${ }^{*}$, Qiuning Zhang ${ }^{1,2,3}$, Hongtao Luo ${ }^{1,2,3}$, Shihong Wei ${ }^{4}$, Tingting Liu ${ }^{4}$, \\ Shilong Sun ${ }^{1,2,3}$, Zhiqiang Liu1,2,3, Zheng Li1,2,3, Jinhui Tian ${ }^{5}$, Xiaohu Wang, $1,2,3 \bowtie$ \\ 1. Institute of Modern Physics, Chinese Academy of Sciences, Lanzhou R.P China, 730000. \\ 2. Lanzhou Heavy Ion Hospital, Lanzhou R.P China, 730000. \\ 3. University of Chinese Academy of Sciences, Beijing R.P China, 100049. \\ 4. Gansu Provincial Cancer Hospital, Lanzhou R.P China, 730050. \\ 5. Center of Evidence Based Medicine, Lanzhou University, Lanzhou R.P China, 730000. \\ ${ }^{*}$ Co-first authors with equal contributions to this work. \\ $\triangle$ Corresponding author: Xiaohu Wang, Tel.: +8613909407551; E-mail: xhwang@impcas.ac.cn; No. 2, Xiaoxihu east street, Qilihe district, Lanzhou city, Gansu \\ province. \\ (c) The author(s). This is an open access article distributed under the terms of the Creative Commons Attribution License (https://creativecommons.org/licenses/by/4.0/). \\ See http://ivyspring.com/terms for full terms and conditions.
}

Received: 2020.06.30; Accepted: 2021.02.03; Published: 2021.03.31

\begin{abstract}
Background: To evaluate locoregional failure and its impact on survival by comparing involved field irradiation (IFI) with elective lymph node irradiation (ENI) for patients with esophageal squamous cell cancer who underwent post-operative radiotherapy.

Methods and Materials: The enrolled patients were randomized allocated to IFI or ENI group. CTV of IFI was generated according to pre-operative primary tumor location and post-operative pathological characters and positive LNs regions. CTV of ENI was generated according to pre-operative tumor position to administer selective lymph node irradiation. Radiotherapy planning was delivered using either 3D-CRT or IMRT.

Results: A total of 57 patients were enrolled, 28 patients in ENI group and 29 patients in IFI group. There were not statistical differences between two groups in baseline $(p>0.05)$. The initial locoregional failure rate was $17.9 \%$ in ENI arm and $20.7 \%$ in IFI arm respectively $(p=0.085)$. The 1-, 3-, and 5-year Progression-free Survival (PFS) were 63.2, 43.5, and $21.8 \%$ in ENI arm versus $78.2,60.1$, and $55.1 \%$ in IFI $\operatorname{arm}(p=0.038)$. The 1-, 3-, and 5 -year overall survival (OS) were $78.6,46.9$, and $23.5 \%$ in ENI arm versus $72.9,59.7$, and $54.3 \%$ in IFI arm $(p=0.06)$. Acute radiation pneumonitis $(p=0.005)$ and hematological toxicities $(p=0.029)$ also showed statistical differences between groups, ENI arm was more than IFI arm.

Conclusions: The results indicated that IFI tended to improve survival and reduce toxicities for patients with operative ESCC and did not increase locoregional failure compared to ENI. It is thus suggested that IFI for ESCC PORT is worthy of clinical recommendation and further study.
\end{abstract}

Key words: esophageal cancer; lymph node; radiotherapy; clinical outcomes; adjuvant treatment

\section{Introduction}

Esophageal cancer $(\mathrm{EC})$ is the $8^{\text {th }}$ most common cancer worldwide. In China, an estimated 320,800 new cases resulted in 253,800 deaths from the disease in 2018 [1,2]. Esophageal squamous cell carcinoma (ESCC) is the main histological subtype in Asian countries. However, in recent decades, the incidence of esophageal adenocarcinoma (EAC) has been increasing in the West $[3,4,5]$. Although surgery is currently the mainstay of treatment in the early stage ESCC and as an important component of 
multimodality treatment in locally advanced ESCC, it exhibits modest efficacy, with a 5-year survival rate of less than $30 \%$ [6]. Approximately half of patients present with locally advanced or metastatic disease at initial diagnosis, and over a third develop regional recurrences or distant metastases after surgery. These results have led to the development of multimodality combination therapy, which includes surgery, chemotherapy, and radiotherapy.

The current National Comprehensive Cancer Network (NCCN) guidelines recommend neoadjuvant or definitive chemoradiation for locally advanced EC [7]. However, these recommendations are often not implemented in clinical practice. In patients with locally advanced ESCC, curative surgery is usually performed at initial diagnosis rather than after neoadjuvant chemoradiotherapy. These patients need to undergo subsequent adjuvant radiotherapy in order to reduce locoregional recurrence and improve progression-free survival (PFS) and overall survival (OS). A review of pertinent literature reveals reports on several non-randomized trials, some of which have evaluated the survival benefit of post-operative radiotherapy (PORT) with inconsistent results. There has been much debate with respect to $\mathrm{pN} 0$ patients, many early studies reported a lack of clinical benefit. However, many recent studies concluded that it was beneficial to $\mathrm{pN} 0$ patients $[8,9,10]$. Sure, the value of PORT is established in patients with pathologically confirmed positive lymph nodes [11]. One of the major reasons for the controversy on clinical benefit was the lack of consensus on the design of an optimal radiation target volume. In cases of locally advanced EC, the use of radical involved field irradiation (IFI) has become increasingly popular among radiation oncologists. Compared to elective nodal irradiation (ENI), nodal failure rates are not higher with IFI, but esophageal toxicities are lower [12]. A meta-analysis including 10 studies with a total of 1348 patients compared the relative advantages and disadvantages of ENI and IFI for the definitive radiotherapy of ESCC. No significant differences in local control or OS were noted between ENI and IFI, and the incidences of zgrade 3 acute esophagitis and pneumonitis were significantly lower in the IFI group. In addition, the use of IFI did not increase the incidence of out-of-field recurrences or metastases. These results generated interest in the use of IFI for PORT in ESCC $[13,14]$.

Up to now, no studies have compared postoperative IFI and ENI in patients with operable ESCC. To address this issue, we performed a preliminary prospective single center randomized controlled study on PORT to investigate the advantages and disadvantages of using IFI and ENI on survival and toxicities in ESCC. The findings are expected to provide guidance for clinical practice.

\section{Materials and Methods}

\section{Patient population}

From January 2012 to December 2018, we conducted a randomized controlled trial in the Gansu Provincial Cancer Hospital to compare IFI and ENI in patients undergoing PORT for ESCC. The inclusion criteria was as follows: 1) patients older than 18 at diagnosis, 2) Karnofsky performance status (KPS) score $\geq 70$, 3) pathologically confirmed ESCC, 4) surgical resection followed by radiotherapy (RT) or chemoradiotherapy (CRT), 5) R1 or R2 resection with any stage, node positive or pT2-4a, 6) radiotherapy delivered using either three-dimensional conformal radiotherapy (3D-CRT) or intensity modulated radiation therapy (IMRT) techniques. The exclusion criteria were as follows: 1) non-ESCC patients, 2) recurrence or metastasis prior to radiotherapy, 3) incomplete RT schedules, and 4) incomplete data on outcomes. Prior to patient enrollment, the study had been approved by the ethics committee of the Gansu Provincial Cancer Hospital (Approval number: A2013112260042). Randomization was performed by computer generated random numbers before initiation of the trial in January 2012. Allocation concealment was implemented on the patients after informed consent. Sample size calculation was based on a margin of non-inferiority for median relapse-free survival time was 11 months that was estimated from previous studies [15]. We found that at least 57 subjects were required in each group in order to obtain a power of $90 \%$ and a two-sided a-level of 0.05 to demonstrate the non-inferiority of IFI with ENI. However, in view of the slow enrollment process, only total 60 subjects were enrolled from January 2012 to June 2018, 3 of including patients with discontinued radiotherapy after enrollment. Finally, 57 patients with complete data, including 28 and 29 patients in the IFI and ENI groups, respectively, were analyzed.

\section{Radiotherapy target volume in patients undergoing ENI}

In patients undergoing ENI, the clinical target volume (CTV) was generated according to the pre-operative tumor position to administer selective node irradiation.

\section{CTV of the upper thoracic segment}

The upper boundary was at the level of cricothyroid membrane, and the lower boundary was at a level of $3 \mathrm{~cm}$ below the tracheal carina. Generally, the target volume incorporates the corresponding areas of lymphatic drainage, the anastomosis, and the 
primary esophageal tumor bed. The inferior cervical, supraclavicular, and mediastinal areas 2, 4, and 7 lymph node (LN) regions should be included.

\section{CTV of the middle thoracic segment}

The upper boundary was at the level of cricothyroid membrane, and the lower boundary was at a level of $3-5 \mathrm{~cm}$ below the preoperative tumor bed. In practice, the target volume should include the corresponding areas of lymphatic drainage, the anastomosis, and the primary esophageal tumor bed. Therefore, bilateral cervical, para-esophageal, lower cervical, supraclavicular, and mediastinal areas 2, 4, 7, and $8 \mathrm{LN}$ regions should be included.

\section{CTV of the lower thoracic segment}

The upper boundary was at the level of the thoracic inlet, and the lower boundary was at a level of 3-5 $\mathrm{cm}$ below the tumor bed. The delineation of the target volume should include the corresponding areas of lymphatic drainage, the anastomosis, and the primary esophageal tumor bed. Therefore, the mediastinal areas 2, 4, 7, and $8 \mathrm{LN}$ regions should be included, but the paracardial and left gastric arterial LN regions are not included unless they are either pathologically positive or enlarged on computed tomography (CT) images.

The LN gross tumor volume (GTVn) was defined as the mediastinal LNs with a diameter of at least $1 \mathrm{~cm}$ in the short axis, or more than $5 \mathrm{~mm}$ in the short axis in the paraesophageal region, tracheoesophageal sulcus, and diaphragmatic angle. Multiple clusters of LNs on the CT image, areas of increased uptake on fluorodeoxyglucose-positron emission tomography (FDG-PET) (excluding physiological accumulation), and pathologically confirmed residual disease were also included in the GTV. The planning target volume (PTV) was then generated by applying $5 \mathrm{~mm}$ radial and $10 \mathrm{~mm}$ longitudinal margins to the $\mathrm{CTV}$, respectively. In those undergoing ENI, a dose of 50-50.4 Gy in 1.8-2 Gy per fraction over 5-5.6 weeks was delivered to the CTV. The GTVs were boosted to a dose of 60 Gy (see Fig. 1A).

\section{Radiotherapy target volume in patients undergoing IFI}

The CTV was delineated based on the preoperative location of the primary tumor and the postoperative extent of pathological invasion, including positive LN regions. Radial and longitudinal margins of 5 and $20 \mathrm{~mm}$, respectively, were added to the tumor bed and surrounding area of lymphatic drainage; positive $\mathrm{LN}$ regions also were included regardless of the location of the primary tumor. Anastomoses were not included unless the margins were positive. The GTVn was similar to that of the ENI group. The PTV was generated by applying $5 \mathrm{~mm}$ radial and $10 \mathrm{~mm}$ longitudinal margins to the CTV. Patients in the IFI arm received a dose of 50-50.4 Gy, delivered in 1.8-2 Gy per fraction over 5-5.6 weeks. The GTV was boosted to at least $60 \mathrm{~Gy}$. The mediastinal LN regions were not electively irradiated in this group (see Fig. 1B).

\section{Treatment planning evaluation and organ-at-risk (OAR) limitations}

Radiotherapy planning was delivered with 6-10 MV photons using Elekta Synergy linear accelerator with either 3D-CRT or IMRT precision radiotherapy
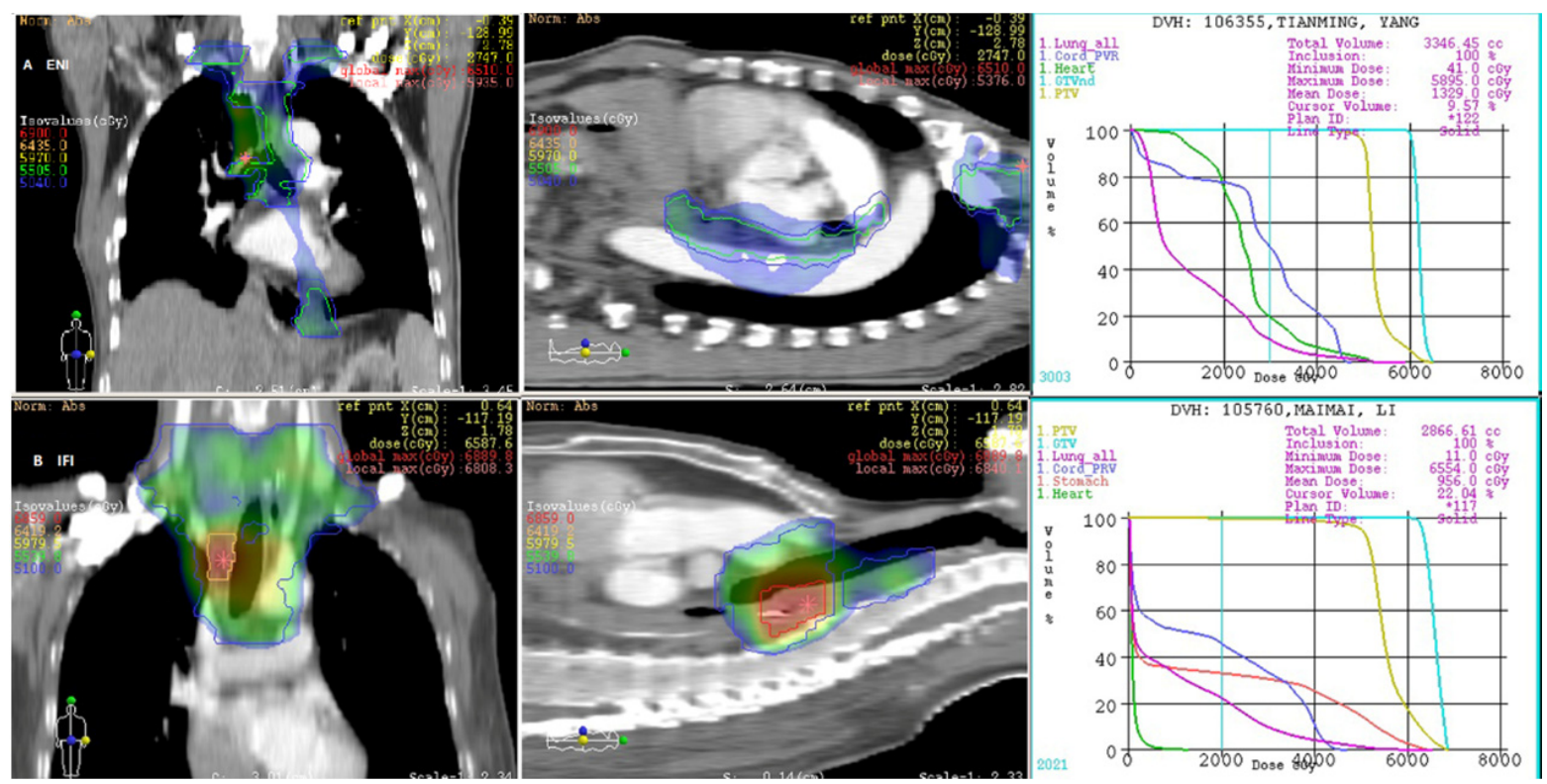

Figure 1. Irradiation field of postoperative radiotherapy using ENI or IFI for 2 patients with upper thoracic esophageal carcinoma. 
technique. The dose homogeneity criteria ensured that the respective PTV-min exceeded $90 \%$ of the prescribed dose, and PTV more than $110 \%$ of the prescribed dose was below $5 \%$. The mean lung dose (MLD) was restricted at or below $15 \mathrm{~Gy}$; in terms of lung volume doses, V20 (the lung volume receiving over $20 \mathrm{~Gy})<30 \%$ or $\mathrm{V} 30<20 \%$ was maintained. Maximum dose of spinal cord was restricted at or below $45 \mathrm{~Gy}$. The maximum dose to the intrathoracic stomach was restricted to below $60 \mathrm{~Gy}$; alternatively, $\mathrm{V} 40<50 \%$ was maintained [16].

\section{Chemotherapy}

Neoadjuvant chemotherapy regimens were not specified before enrollment. However, subgroup analysis was performed while summarizing data. Concomitant chemotherapy was not administered during adjuvant radiotherapy. All patients received 2-4 cycles of adjuvant chemotherapy following completion of RT. Chemotherapy regimens comprised fluorouracil (5-FU) or docetaxel (TXT) doublets including either cisplatin (DDP) or nedaplatin (NDP). In patients aged 75 years or older, dose reductions to $80 \%$ of the dose were made. Adjuvant chemotherapy was initiated 2-4 weeks after either surgery or PORT.

\section{Data collection}

The primary endpoints were the initial locoregional failure rate and the incidence of toxicity. The secondary endpoints were PFS, OS, and dosimetry parameters. The variables collected for each patient included baseline characteristics (age and sex), pre-radiotherapy KPS score, characteristics of the tumor (primary site, extent of lesion, stage of tumor, histology, grade, and nodal status), type of surgery, status of surgical margins, interval between surgery and RT, extent of irradiation field, RT technique, RT dosimetry parameters (total RT dose, target volume, and OAR dose), chemotherapy agents, chemotherapy cycles, date of recurrence or progression, and date of death. All the relevant data were obtained from the hospital records.

\section{Statistical analyses}

The constituent ratio was analyzed by the chisquare test, and the measurement data were analyzed by the nonparametric test. The Kaplan-Meier method with the log-rank test was employed to estimate differences in 1-, 3-, and 5-year PFS and OS between patients receiving IFI and ENI. The t-test was used to compare the dosimetry differences. All $p$ values were based on a 2-sided test, and the differences were regarded as statistically significant when $p<0.05$. All statistical analyses were performed using the SPSS (Version 22.0) statistical software package.

\section{Follow-up and evaluation criteria}

All patients who completed treatment were routinely followed-up every 3 months in the first 2 years, every 6 months in the third to fifth year, and every 12 months thereafter. Follow-up routinely included physical examination, laboratory examination, CT scans of the thorax and upper abdomen, and ultrasound examination of the cervical LNs. Upper gastrointestinal endoscopy (with or without biopsy) was not performed unless indicated. CT scan of the thorax and upper abdomen was performed at each visit, and PET/CT was performed every 6-12 months or in cases of suspected recurrence.

\section{Results}

\section{Patient characteristics}

Till date, 57 patients with ESCC who underwent esophagectomy have been enrolled in this trial. The characteristics of the 57 eligible patients are shown in Table 1. Gender, age, performance status, cancer staging, tumor extent and location, surgical procedures, LN dissection rate, RT techniques, interval between surgery and RT, and cycles of neoadjuvant chemotherapy were not statistical differences between two groups $(p>0.05)$. However, the cycles of adjuvant chemotherapy differed significantly between the two groups $(p=0.031)$ (see Table 1).

Table 1. Patients' clinical characteristic

\begin{tabular}{|c|c|c|c|c|}
\hline Factor & ENI n $(\%)$ & IFI n (\%) & $X^{2}\left({ }^{*} \mathrm{~F}\right.$ value $)$ & $P$ value \\
\hline \multicolumn{5}{|l|}{ Total } \\
\hline Subgroup & 28 & 29 & & \\
\hline \multicolumn{5}{|l|}{ Sex } \\
\hline Female & $2(3.5)$ & $1(1.7)$ & 0.390 & 0.532 \\
\hline Male & $26(45.6)$ & $28(49.2)$ & & \\
\hline \multicolumn{5}{|l|}{ Age (y.o.) } \\
\hline Range & $40-69$ & $46-76$ & $0.016^{*}$ & 0.900 \\
\hline Median & $58.5 \pm 8.7$ & $58.7 \pm 8.6$ & & \\
\hline \multicolumn{5}{|l|}{ ECOG } \\
\hline 1 & $8(14.0)$ & $13(22.8)$ & 3.292 & 0.193 \\
\hline 2 & 18 (31.6) & $16(28.1)$ & & \\
\hline 3 & $2(3.5)$ & 0 & & \\
\hline \multicolumn{5}{|l|}{ Location } \\
\hline Ut & $2(3.5)$ & $4(7.0 \%)$ & 0.697 & 0.706 \\
\hline Mt & $15(26.3)$ & $15(26.3)$ & & \\
\hline Lt & $11(19.4)$ & $10(17.5)$ & & \\
\hline \multicolumn{5}{|c|}{ Lesion length (cm) } \\
\hline Range & $3-10$ & $3-7$ & $1.524^{*}$ & 0.222 \\
\hline Median & $5.3 \pm 1.6$ & $4.5 \pm 1.1$ & & \\
\hline \multicolumn{5}{|c|}{ Surgical procedure } \\
\hline Single incision & $15(26.3)$ & $13(22.8)$ & 3.656 & 0.161 \\
\hline Two incisions & $10(17.5)$ & $7(12.3)$ & & \\
\hline Three incisions & $3(5.3)$ & $9(15.8)$ & & \\
\hline \multicolumn{5}{|l|}{ Clinical stage } \\
\hline I & 0 & $3(5.3)$ & 5.130 & 0.163 \\
\hline II & $11(19.3)$ & $7(12.3)$ & & \\
\hline III & $17(29.8)$ & $19(33.3)$ & & \\
\hline Lymph node di & & & & \\
\hline
\end{tabular}




\begin{tabular}{|c|c|c|c|c|}
\hline Factor & ENI n (\%) & IFI n (\%) & $X^{2}\left({ }^{*} \mathrm{~F}\right.$ value $)$ & $P$ value \\
\hline No. of positive & $2.5 \pm 2.9$ & $2.3 \pm 3.7$ & $0.229^{*}$ & 0.634 \\
\hline No. of total & $16.5 \pm 8.5$ & $14.8 \pm 8.8$ & $0.000^{*}$ & 0.997 \\
\hline \multicolumn{5}{|l|}{ RT technology } \\
\hline 3D-CRT & $7(12.3)$ & $10(17.5)$ & 0.612 & 0.434 \\
\hline IMRT & $21(36.8)$ & $19(33.3)$ & & \\
\hline \multicolumn{5}{|c|}{ Interval between surgery to RT (mo) } \\
\hline Range & $0.9-10$ & $0.6-10.9$ & $0.221^{*}$ & 0.640 \\
\hline Median & $3.9 \pm 1.9$ & $2.7 \pm 1.9$ & & \\
\hline Pts of neoadjuvant chemo & $4(14.3)$ & $6(20.7)$ & & \\
\hline No. of cycles & $1.7 \pm 0.5$ & $2.3 \pm 1.9$ & $1.610^{*}$ & 0.240 \\
\hline Pts of adjuvant chemo & $24(85.7)$ & $22(75.9)$ & & \\
\hline No. of cycles & $3.1 \pm 1.4$ & $2.4 \pm 1.0$ & $4.995^{*}$ & 0.031 \\
\hline \multicolumn{5}{|l|}{ Follow-up time(mo) } \\
\hline Range & $2.7-78.8$ & $8.9-65.6$ & $0.185^{*}$ & 0.669 \\
\hline Median & $28.5 \pm 21.7$ & $34.6 \pm 19.2$ & & \\
\hline
\end{tabular}

Note: * $\mathrm{F}$ value in $\mathrm{T}$ test.

Abbreviations: ENI, elective lymph node irradiation; IFI, involved field irradiation; y.o., years old; mo, months; RT, radiotherapy; Pts, patients; Chemo, chemotherapy; No., numbers.

Table 2. Treatment results between ENI and IFI

\begin{tabular}{|c|c|c|c|c|}
\hline Factors & ENI n (\%) & IFI n (\%) & $\chi^{2}$ & $P$ value \\
\hline \multicolumn{5}{|l|}{ Total } \\
\hline Subgroup & 28 & 29 & & \\
\hline \multicolumn{5}{|l|}{ State at analysis } \\
\hline Alive & $11(39.3)$ & $18(62.1)$ & 2.959 & 0.085 \\
\hline Dead & $17(60.7)$ & $11(37.9)$ & & \\
\hline \multicolumn{5}{|l|}{ Cause of death } \\
\hline Cancer related & $12(42.9)$ & $8(28.5)$ & 0.15 & 0.903 \\
\hline Non-cancer related & $5(17.9)$ & $3(10.7)$ & & \\
\hline First locoregional failure & $5(17.9)$ & $6(20.7)$ & 0.073 & 0.786 \\
\hline Primary tumor & $4(14.3)$ & $2(6.9)$ & & \\
\hline Regional lymph node & $1(3.6)$ & $4(13.8)$ & & \\
\hline First distance metastasis & $7(25.0)$ & $7(24.1)$ & 0.006 & 0.940 \\
\hline Lymph node & $2(7.1)$ & $3(10.7)$ & & \\
\hline Lung & $2(7.1)$ & $2(6.9)$ & & \\
\hline Liver & $1(3.6)$ & $1(3.4)$ & & \\
\hline Bone & $2(7.2)$ & $1(3.4)$ & & \\
\hline \multicolumn{5}{|l|}{ PFS } \\
\hline 1-year & $18(63.2)$ & $23(78.2)$ & 1.593 & 0.207 \\
\hline 3-year & $12(43.5)$ & $17(60.1)$ & 1.416 & 0.234 \\
\hline 5-year & $6(21.8)$ & $16(55.1)$ & 6.844 & 0.009 \\
\hline \multicolumn{5}{|l|}{ OS } \\
\hline 1-year & $22(78.6)$ & $21(72.9 \%)$ & 0.292 & 0.589 \\
\hline 3-year & $13(46.9)$ & $17(59.7 \%)$ & 0.849 & 0.357 \\
\hline 5-year & $7(23.5)$ & $16(54.3 \%)$ & 5.388 & 0.020 \\
\hline
\end{tabular}

\section{Locoregional failure, distant metastases, and survival}

Locoregional failure includes recurrence at the site of the primary tumor and regional LN progression. Overall, $5(17.9 \%)$ and $6(20.7 \%)$ patients in the ENI and IFI groups had initial locoregional failure, respectively $(p=0.085)$. Among them, $4(14.3 \%)$ and $2(6.9 \%)$ patients experienced recurrence of the primary tumor, while $1(3.6 \%)$ and $4(13.8 \%)$ patients experienced progression in the regional LNs in the ENI and IFI groups, respectively. Initial distant metastases were observed in 7 (25\%) and 7 (24.1\%) patients in the ENI and IFI groups, respectively (see
Table 2).

At the time of analysis, 29 patients, comprising $11(39.3 \%)$ and $18(62.1 \%)$ patients in the ENI and IFI groups, respectively, were alive. It should be noted that there is very small statistical difference was observed between the groups $(p=0.085)$. The respective 1-, 3-, and 5-year PFS rates were $63.2 \%$, $43.5 \%$, and $21.8 \%$ in ENI group and $78.2 \%, 60.1 \%$, and $55.1 \%$ in IFI group, ( $p=0.038$ by the log-rank test) as shown in Fig. 2, there was statistical difference in 5 -year PFS $(p=0.009)$. The median PFS was $17.5(95 \%$ CI: 5.3-42.9) months versus 37.1 (95\% CI: 19.2-54.9) months in the ENI and IFI groups, respectively. Furthermore, the PFS was significantly superior in the IFI group than that of the ENI group.

The median survival time in the ENI and IFI groups was 36.3 (95\% CI: 13.8-58.9) months versus 42.4 (95\% CI: 30.2-54.6) months, respectively. The respective 1-, 3-, and 5-year overall survival (OS) rates in the ENI and IFI groups were $78.6 \%, 46.9 \%$, and $23.5 \%$ versus $72.9 \%, 59.7 \%$, and 54.3 ( $p=0.06$ by the log-rank test), as shown in Fig. 3, there was statistically difference in 5 -year OS $(p=0.020)$. The respective overall 1-, 3-, and 5-year OS rates were $100 \%$ each in stage $\mathrm{I}, 85.8 \%, 51.8 \%$, and $34.6 \%$ in stage II, and $77.8 \%, 47.9 \%$, and $34.2 \%$ in stage III, respectively ( $p=0.0001)$ (Fig. 4$)$.

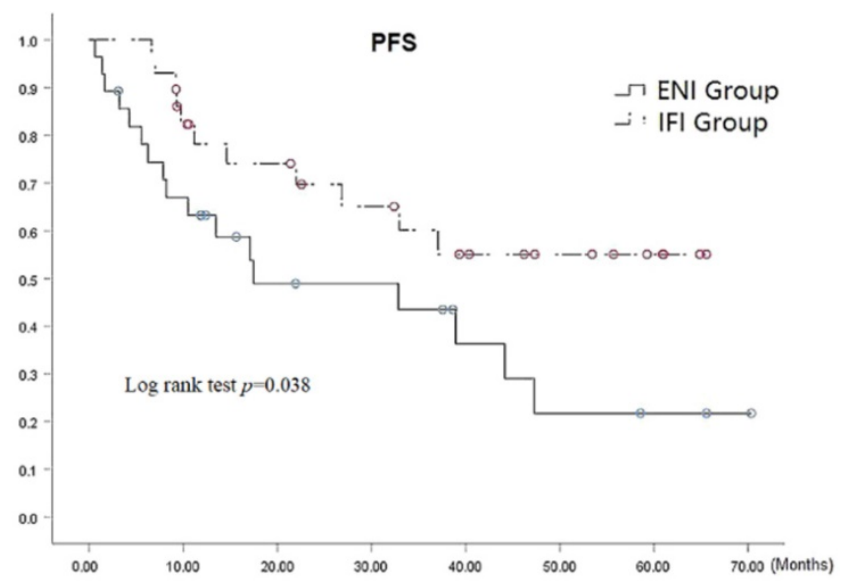

Figure 2. PFS Curves for patients with ENI vs. IFI.

\section{Acute and late toxicities}

Acute and late radiation induced toxicities were evaluated based on the Radiation Therapy Oncology Group (RTOG) toxicity criteria [17]. Wilcoxon rank sum tests were used to compare the incidence of toxicities between the two groups, by grade. The incidences of acute radiation pneumonitis $(p=0.005)$ and hematological toxicities $(p=0.029)$ were significantly higher in the ENI group than in those receiving IFI. No statistical differences were observed in terms of acute radiation-induced esophagitis 
( $p=0.344)$, acute digestive toxicities $(p=0.428)$, late esophageal stenosis $(p=0.110)$, pulmonary interstitial fibrosis, $(p=0.055)$, and late heart injury $(p=0.309)$ between two groups. We compared toxicities of grades 3-5 as severe toxicity using the Chi-square test. Higher than grade 3 acute radiation-induced esophagitis, acute radiation pneumonitis, hematological toxicities, acute digestive toxicities, late esophageal injury, and late lung injury were observed in $4(10.3 \%), 1(3.6 \%), 8(28.6 \%), 1(3.6 \%)$ and $1(3.6 \%)$ patients, respectively, in the ENI group, and in 1 $(3.4 \%), 1$ (3.4\%), 3 (10.3\%), 1 (3.4\%) and $1(3.4 \%)$ patients in the IFI group (see Table 3). Notably, all 3 patients with grade 5 toxicity in the ENI group had following issues, who died of esophageal perforation, upper gastrointestinal bleeding, and esophagotracheal fistula, respectively.

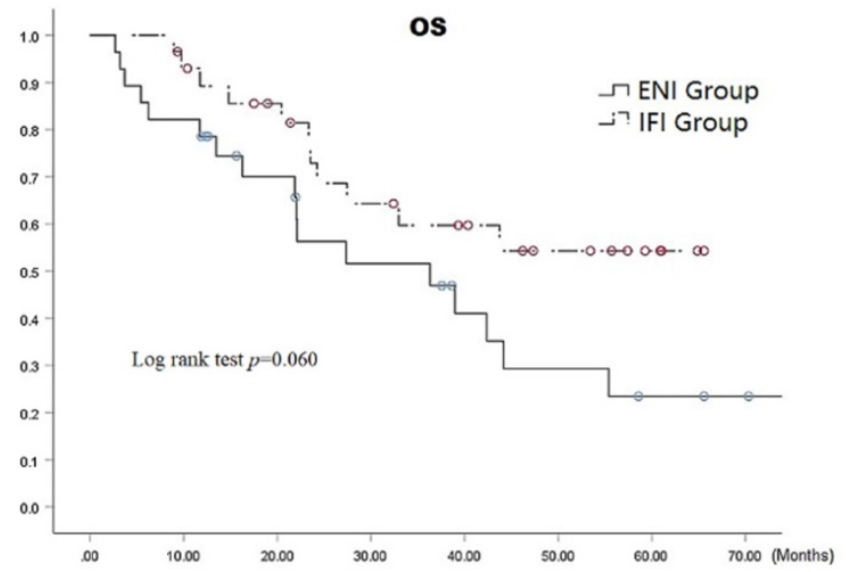

Figure 3. OS with Stage Curves for all patients.

Table 3. Comparison of grade 3-5 acute and late radiated adverse events between two groups

\begin{tabular}{llllll}
\hline Factors & Grade & ENI (n\%) & IFI (n\%) & $X^{2}$ & $P$ value \\
\hline $\begin{array}{l}\text { Acute radiation- } \\
\text { induced esophagitis }\end{array}$ & Grade 3 & $2(7.1 \%)$ & $0(0 \%)$ & 2.091 & 0.148 \\
& Grade 4 & $1(3.6 \%)$ & $1(3.4 \%)$ & & \\
Acute radiation & Grade 5 & $1(3.6 \%)$ & 0 & & \\
$\begin{array}{l}\text { pneumonitis } \\
\text { Hematological toxicities } 3\end{array}$ & $1(3.6 \%)$ & $1(3.4 \%)$ & 0.001 & 0.980 \\
$\begin{array}{l}\text { Acute digestive } \\
\text { toxicities }\end{array}$ & Grade 3 & $8(28.6 \%)$ & $3(10.3 \%)$ & 3.039 & 0.081 \\
$\begin{array}{l}\text { Late esophageal } 3 \\
\text { stenosis }\end{array}$ & Grade 5 & $1(7.1 \%)$ & $1(3.4 \%)$ & 1.002 & 0.317 \\
$\begin{array}{l}\text { Pulmonary interstitial } \\
\text { fibrosis }\end{array}$ & Grade 4 & $0(0 \%)$ & 0 & & \\
\hline
\end{tabular}

\section{Dosimetric evaluation}

Dose volume histograms were used to calculate the dosimetry parameters. There were statistical differences between the groups in terms of PTV $(p=0.014)$, GTV $(p=0.038)$, maximum dose of spinal cord $(p=0.024)$, and MLD ( $p=0.049), \mathrm{V} 5(p=0.005), \mathrm{V} 10$ $(p=0.042), \mathrm{V} 20(p=0.018), \mathrm{V} 30(p=0.035)$ of the lungs, the ENI group demonstrated higher values than the IFI group. No statistical differences were noted between the two groups in terms of mean PTV and GTV doses, maximum dose to the intrathoracic stomach, and V30 of the heart; $p>0.05$ (see Table 4).

\section{Discussion}

This study aimed to compare the differences in locoregional failure, survival, toxicities and dosimetry between ENI and IFI for patients receiving PORT with operable ESCC. Since it was proposed in the 1960s, the value of PORT in EC has been widely reported. A large randomized controlled clinical trial from China further confirmed the value of PORT in ESCC [18]. In that study, 495 patients who has been through the radical surgery for ESCC were randomized allocated by the envelope method to receive surgery $(n=275)$ or surgery with radiotherapy $(n=220)$; the findings showed that prophylactic PORT improved survival in patients with stage III tumors who underwent radical resection (5-year OS: $13.1 \%$ vs. $35.1 \%$, respectively) and reduced the incidence of failure in the intra-thoracic LNs and anastomosis (19.8\% vs. $44.9 \%)$, without any increase in the incidence of anastomotic strictures. Nevertheless, many earlier randomized controlled trials demonstrated inconsistent results. For instance, Fok et al. and Ténière et al. reported no difference in survival among patients receiving PORT or surgery alone $[19,20]$. However, in interpreting these findings, it is important to note that the aforementioned results in the era of two-dimensional RT planning, when treatment was delivered using non-conformal anteroposterior-posteroanterior fields. Advanced RT techniques had not emerged, and the treatment fields were usually more extensive. Therefore, these findings from the early 1990s are not representative of current treatment outcomes. It was therefore essential to re-evaluate the influence of PORT fields on OS and local-regional recurrence in the era of precision RT. Although there are some studies on the extent of RT fields, most of them have focused on radical radiotherapy instead of PORT [21]. Till date, two meta-analyses have compared IFI with ENI for radical CRT in EC. The results have shown no significant differences between those receiving IFI and ENI, in terms of OS and local control [22,23]. However, the incidence of esophageal and lung toxicities was significantly lower in those receiving IFI. These results show that IFI is a viable treatment option in locally advanced EC, and is particularly useful in minimizing radiation-related toxicities. To the best of our knowledge, no prospective studies have compared ENI with IFI in patients receiving PORT for EC. 


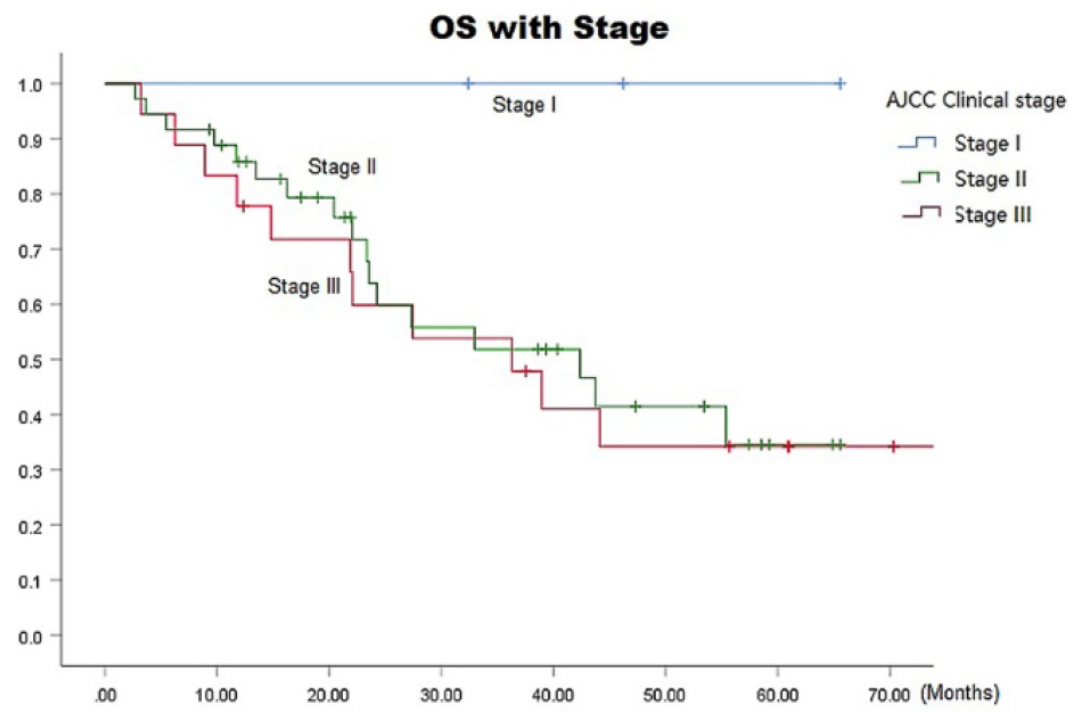

Figure 4. OS Curves for patients with ENI vs. IFI.

There is currently no consensus on the optimal CTV extent for PORT in EC. Usually, the target volumes for PORT depend on the following factors: 1 ) stage and location of the primary lesion; 2 ) major sites of relapse; 3) and the area of surgical non-clearance. However, owing to nodal skip metastases and inconsistencies in surgical procedures, it is often difficult to evaluate the exact major sites of relapse, and the areas of surgical non-clearance. Therefore, the NCCN guidelines suggest that the standard PORT CTV should encompass the tumor bed, bilateral supraclavicular LN areas, mediastinal LNs, and the LNs around the cardia and left gastric artery [8]. It appears that the guideline has ignored the individual characteristics of patients with ESCC. Since the recommended extent of the PORT surpass that of radical radiotherapy, this issue deserves serious consideration. Some studies found the probability of skip metastases in EC to be only 5\%-10\% [17,24]. This may allow scope for optimization of irradiation fields to reduce adverse events. Previous studies have provided several suggestions for the extent of the CTV of PORT in ESCC. These include: 1) bilateral supraclavicular areas from the level of the Adam's apple to the subclavian artery, the entire mediastinum, and the left gastric lymphatic area $[17,25], 2)$ bilateral supraclavicular regions, entire mediastinum, and the left gastric region only if it was involved [20], 3) tumor bed only, and bilateral supraclavicular and left gastric lymphatic areas for lesions in the upper and lower thoracic segments, respectively [26], 4) margins of $3-4 \mathrm{~cm}$ around the tumor bed in the cranio-caudal direction, without inclusion of left gastric and bilateral supraclavicular areas for prophylactic irradiation [27], 5) T-shaped fields including bilateral supraclavicular and upper mediastinal areas [28]. Despite many attempts to minimize the PORT field, the exact coverage of high-risk areas was difficult to achieve with two-dimensional radiotherapy.

The study has found that the incidence of local lymph node metastasis is related to the site of the primary tumor [29]. For example, upper thoracic esophagus tumor often metastasizes to cervical lymph nodes, while lower thoracic tumor are more likely to metastasizes to upper abdominal lymph nodes. Therefore, there may be a special relationship between the tumor location and the recurrence of $\mathrm{LN}$. In view of these findings, ENI fields were usually the most widely accepted for adequate CTV coverage for either radical radiotherapy or PORT. However, the use of IFI has become increasingly popular in the treatment of locally advanced EC. In IFI, the CTV is usually generated by adding no radial margins and but only adding $2 \mathrm{~cm}$ longitudinal margins to the GTVprimary; no margins are added for the GTV-LNs, and the mediastinal LNs are not electively irradiated, only involved LN areas, diagnosed by imaging, are irradiated [23,24]. This method of accurate CTV delineation was made possible by the advent of precision radiotherapy and by improvements in imaging. IMRT involves computer-based planning; it has improved target volume coverage in EC with less off-target delivery, potentially reducing toxicities and allowing further dose escalation beyond the limits of 3D-CRT [30]. Therefore, if advanced radiotherapy techniques and imaging are employed, IFI may be feasible for PORT in ESCC. In this study, we re-defined the PORT fields as suggested by the expert consensus and recommendations for target volume delineation in the NCCN guideline $[8,28,31,32,33]$. In our study, the CTV for IFI was generated according to the pre-operative location of the primary tumor and the post-operative extent of pathological invasion and positive LNs regions. The CTV included $5 \mathrm{~mm}$ radial and $20 \mathrm{~mm}$ longitudinal margins, respectively, to the tumor bed and positive LN regions; the anastomosis was not included unless the margin was positive. The implementation of this PORT field necessitates the use of standardized surgical procedures for radical esophagectomy. These include either a three- or twofield lymphadenectomy, since recent studies have demonstrated no obvious differences in impact of either approach on the PORT CTV $[34,35]$. 
Table 4. Comparison of dose distribution parameters for tumor target volume and OAR between ENI Group and INI Group

\begin{tabular}{|c|c|c|c|c|c|c|c|c|c|c|c|c|c|}
\hline Item & $\begin{array}{l}\text { PTV volume } \\
\left(\mathrm{cm}^{3}\right)\end{array}$ & $\begin{array}{l}\text { GTV volume } \\
\left(\mathrm{cm}^{3}\right)\end{array}$ & $\begin{array}{l}\text { PTV Dmean } \\
\text { (Gy) }\end{array}$ & $\begin{array}{l}\text { GTV Dmean } \\
\text { (Gy) }\end{array}$ & $\begin{array}{l}\text { Lung volume } \\
\left(\mathrm{cm}^{3}\right)\end{array}$ & $\begin{array}{l}\text { MLD } \\
\text { (Gy) }\end{array}$ & $\begin{array}{l}\text { Lung V5 } \\
(\%)\end{array}$ & $\begin{array}{l}\text { Lung } \\
\text { V10 (\%) }\end{array}$ & $\begin{array}{l}\text { Lung } \\
\text { V20 (\%) }\end{array}$ & $\begin{array}{l}\text { Lung } \\
\text { V30 (\%) }\end{array}$ & $\begin{array}{l}\text { Thorax stomach } \\
\text { Dmax (Gy) }\end{array}$ & $\begin{array}{l}\text { Heart } \\
\text { V30 (\%) }\end{array}$ & $\begin{array}{l}\text { SP Dmax } \\
\text { (Gy) }\end{array}$ \\
\hline ENI & $353.5 \pm 123.7$ & $47.0 \pm 24.1$ & $49.9 \pm 2.6$ & $60.2 \pm 2.5$ & $3167 \pm 321$ & $13.1 \pm 1.6$ & $55.9 \pm 6.7$ & $43.1 \pm 5.0$ & $27.6 \pm 2.6$ & $18.3 \pm 1.6$ & $54.5 \pm 4.4$ & $41.1 \pm 9.5$ & $43.8 \pm 1.7$ \\
\hline IFI & $210 \pm 82.2$ & $36.1 \pm 13.1$ & $51.0 \pm 3.5$ & $60.5 \pm 1.5$ & $3224 \pm 471$ & $10.8 \pm 0.9$ & $48.7 \pm 4.2$ & $37.9 \pm 3.7$ & $19.9 \pm 2.4$ & $12.0 \pm 2.1$ & $55.9 \pm 4.3$ & $38.0 \pm 8.8$ & $42.4 \pm 2.7$ \\
\hline F value & 0.454 & 4.755 & 1.660 & 1.281 & 3.405 & 4.141 & 8.668 & 4.345 & 5.141 & 3.889 & 0.019 & 0.025 & 5.385 \\
\hline$P$ value & 0.014 & 0.038 & 0.203 & 0.268 & 0.07 & 0.049 & 0.005 & 0.042 & 0.018 & 0.035 & 0.890 & 0.876 & 0.024 \\
\hline
\end{tabular}

Abbreviations: GTV, gross tumor volume; PTV, planning target volume; Dmean, mean dose; Dmax, absolute maximal dose; MLD, mean of lung dose; SP, spinal cord.

In the current study, the 3- and 5-year OS were $59.7 \%$ and $54.3 \%$ in IFI group versus $46.9 \%$ and $23.5 \%$ in ENI group, respectively. Although the difference in OS between the groups was not statistically significant $(p=0.06)$, the absolute survival time in the IFI group was longer. Moreover, the 3- and 5-year PFS were $60.1 \%$ and $55.1 \%$ in IFI group versus $43.5 \%$ and $21.8 \%$ in ENI group, respectively $(p=0.038)$. These results were evidently superior to that of previous reports from randomized trials on ENI. Chen et al. [36] reported a 3- and 5-year OS of $53.1 \%$ and $44.6 \%$, respectively, using $\mathrm{T}$-shaped fields to treat node positive thoracic ESCC in patients who underwent 3-field lymphadenectomy with esophagectomy. Xiao et al. [37] randomized patients with ESCC who underwent radical esophagectomy to receive surgery alone or PORT; the CTV included bilateral supraclavicular regions, the whole mediastinum, and the left gastric arterial LN region. The 5-year OS was $41 \%$ with acceptable toxicities. Wang et al. [38] conducted a randomized controlled clinical trial to explore whether PORT could improve the prognosis in patients with ESCC who were at a high risk of poor clinical outcomes; the 5-year OS was $48.1 \%$. Considering the possibility of longitudinal drainage of submucosal lymphatic network and skip lymph node metastasis in ESCC, the standard PORT CTV should include tumor bed, bilateral supraclavicular region, mediastinal LNs, cardia and left gastric region $[39,40]$. Till date, few studies have employed the exact standard radiation field without suitable adjustments, as serious complications may nullify the survival advantage in certain patients. In our current study, the incidence of acute and late grade 3-5 adverse events in the IFI group was quite low, and there were no fatal toxicities. In addition, the incidence of more than grade 3 acute radiation-induced esophagitis, acute radiation pneumonitis, hematological toxicities, acute digestive toxicities, late esophageal injury and late lung injury only all was only $3.4 \%$ in all patients. Conversely, in the ENI group, the incidence of grade 3-5 acute and late radiation related adverse events were higher, with 3 related deaths. Among them, 1 patient each died of acute esophageal perforation, acute upper gastrointestinal bleeding, and a late esophageal mediastinal fistula, respectively.
The rates of initial locoregional failure and distant metastases were similar in both groups. Notably, till the date of last follow-up, compared to 1 patient in the ENI group, 4 patients in the IFI group had regional LN recurrences. The sites of failure were the commonly reported sites, viz., bilateral supraclavicular areas and the superior mediastinum. The findings suggest that regional lymph node have a high-risk of failure rate in IFI group, particularly in the supraclavicular and the superior mediastinal areas. Further large sample controlled clinical trials are necessary for validation, we should be careful in final conclusion. However, the rates of distant metastases were similar in both groups.

Dosimetry parameter is a very important issue of concern for radiation oncologists. Doses of OARs are of particular interest as these are related to clinical advantages and disadvantages. It is obvious that any reduction of the target volume leads to reductions in the doses of OARs, with consequent lowering of radiation toxicities. We had explored various physics-based improvements for radiotherapy of the entire esophagus and T-shaped field using IMRT. The results had demonstrated that small field IMRT plans provided superior lung and heart sparing compared to larger IMRT fields; the jaw tracking technique provided further normal tissue sparing compared to fixed jaw plans $[41,42,43]$. In the current study, there were statistical differences between the fields in terms of may parameters including PTV, GTV, MLD, V5, $\mathrm{V} 10, \mathrm{~V} 20$, and V30 of the lungs, and the maximum dose to the spinal cord. All of these were higher in the ENI group.

Although preoperative chemoradiotherapy is being increasingly recommended as a preferred treatment model for locally advanced EC, the prevalence and availability of this approach is a matter of concern [44,45]. Most surgeons prefer either preoperative chemotherapy or surgery alone instead of neoadjuvant chemoradiotherapy; this makes post-operative chemoradiotherapy essential for stage II or III ESCC. Sadrizadeh A. et al. compared the benefits of neoadjuvant chemoradiotherapy with that of postoperative chemoradiotherapy (POCRT). The results indicated that neoadjuvant chemoradiotherapy offered no survival advantage over POCRT. 
However, POCRT was associated with a higher risk of postoperative complications [46]. In the current study, adjuvant chemotherapy was administered to $85.7 \%$ and $75.8 \%$ in ENI and IFI groups, respectively; the numbers of chemotherapy cycles were higher in ENI group than that of IFI group (3.1 vs. 2.4); this also could explain the higher incidence of toxicities in the former. In view of the risk of cumulative toxicities from either treatment modality, the PORT field should be delineated with care in cases of POCRT. Several studies have demonstrated that in ESCC, POCRT is significantly more effective than PORT alone. This is particularly applicable for patients with vascular emboli and other poor prognostic factors [47]. Nevertheless, postoperative chemotherapy could safely be added to PORT either using ENI or IFI, and POCRT plays an increasingly important role in the comprehensive treatment of EC.

The current prospective clinical trial has certain limitations. The findings in this study may have been biased owing to significant differences in $\mathrm{T}$ and $\mathrm{N}$ stages, and performance status, between the groups. The IFI group demonstrated superior survival with more patients with $\mathrm{T} 1$ and N1 stages, and good performance status. More patients with N2-3 disease and inferior performance status were in the ENI arm. These may have affected the data on treatment outcomes. In addition, PET-CT was not routinely used in defining viable lymph node metastases; this may have affected the accuracy of target volume delineation. Moreover, multiple factors such as tumor location, POCRT, and TNM stage, among others, influence postoperative recurrence in ESCC.

\section{Conclusion}

In summary, compared to ENI, IFI provided superior survival in this cohort, with less toxicities in patients receiving PORT for ESCC. In addition, IFI did not increase locoregional failure. IFI may be recommended in the clinical practice for PORT in ESCC. Further studies are needed to validate our findings.

\section{Acknowledgements}

This study was supported by Talent innovation and venture project of Lanzhou city (No. 2017-RC-23 and 2020-RC-113), Key R\&D Program of Science and Technology Department of Gansu Province (No. 20YF8FA116), Gansu provincial healthcare industry science research project (No. GSWSKY-2017-17), and National key Research and Development Project of China (No. 2016YFC0105706), the authors wish to thank abovementioned organizations. The authors are grateful for the contributions of all patients who enrolled in this trial. We would like to thanks Kambiz, a Medical Physicist come from USA who worked in SPHIC hospital, he has paid a great effort in the final revision of the manuscript.

\section{Competing Interests}

The authors have declared that no competing interest exists.

\section{References}

1. Chen W, Zheng R, Baade PD, Zhang S, Zeng H, Bray F, et al. Cancer statistics in China, 2015.CA CANCER J CLIN. 2016; 66(2):115-132.

2. Siegel RL, Miller KD, Jemal A. Cancer statistics, 2018. CA Cancer J Clin 2018; 68:7-30

3. Rustgi AK, El-Serag HB. Esophageal carcinoma. N Engl J Med 2014; 371:2499509.

4. Rubenstein $\mathrm{JH}$, Shaheen NJ. Epidemiology, diagnosis, and management of esophageal adenocarcinoma. Gastroenterology 2015; 149:302-17.

5. Wang QL, Xie SH, Wahlin K, Lagergren J. Global time trends in the incidence of esophageal squamous cell carcinoma. Clin Epidemiol 2018; 10:717-728.

6. Thumallapally N, Meshref A, Mousa M, Hendawi M, Lan M, Salem AI, Forte F. Survival benefit of neoadjuvant versus adjuvant radiotherapy in lymph node positive esophageal cancer: a population based analysis. J Gastrointest Oncol 2017; 8(5):825-832. doi: 10.21037/jgo.2017.06.19.

7. [Internet] Esophageal and esophagogastric junction cancers, NCCN guidelines, version 1. 2019. Available online: http:/ / www.nccn.org/.

8. Chen JQ, Pang JJ, Chen MQ, Zhu KS, Li YY, Wang JZ, et al. Postoperative prophylactic radiotherapy for $\mathrm{N} 0$ esophageal squamous cell carcinoma[J]. Zhonghua Fang She Zhong Liu Xue Za Zhi, 2009, 18(4): 261-4.

9. Martin JT, Worni M, Zwischenberger JB, Gloor B, Pietrobon R, D'Amico TA, et al. The role of radiation therapy in resected T2N0 esophageal cancer: a population-based analysis. Ann Thorac Surg, 2013, 95(2): 453-8.

10. Hsu PK, Huang CS, Wang BY, Wu YC, Hsu WH. Survival benefits of postoperative chemoradiation for lymph node-positive esophageal squamous cell carcinoma. Ann Thorac Surg, 2014, 97(5): 1734-41.

11. Shen WB, Zhu SC. Progress of Clinical Application of Radiochemotherapy after Esophagectomy. Cancer Res Prev Treat, 2015, 42(6): 535-40.

12. Yamashita $H$, Takenaka R, Omori M, Imae $T$, Okuma $K$, Ohtomo $K$, et al. Involved-field radiotherapy (IFRT) versus elective nodal irradiation (ENI) in combination with concurrent chemotherapy for 239 esophageal cancers: a single institutional retrospective study. Radiat Oncol. 2015,10(8):171.

13. Kimoto T, Yamazaki H, Suzuki G, Aibe N, Masui K, Tatekawa K, et al. Local field radiotherapy without elective nodal irradiation for postoperative locoregional recurrence of esophageal cancer. Jpn J Clin Oncol.2017;47(9):809-814.

14. Liu J, Cai X, Liu Q, Li H, Cheng Y, Fu X. Characteristics of the local recurrence pattern after curative resection and values in target region delineation in postoperative radiotherapy for lower thoracic esophageal squamous cell cancer. Thorac Cancer. 2017;8(6):630-633.

15. Ku GY, Ilson DH. Adjuvant (postoperative) therapy for esophageal cancer. Thorac Surg Clin. 2013;23(4):525-533.

16. Chinese radiotherapy guidelines for esophageal cancer (2019 edition)[J]. International Journal of Oncology. 2019(07):385-398.

17. Cox JD, Stetz J, Pajak TF. Toxicity criteria of the Radiation Therapy Oncology Group (RTOG) and the European Organization for Research and Treatment of cancer (EORTC). Int J Radiat Oncol Biol Phys, 1995, 31(5): 1341.

18. Xiao ZF, Yang ZY, Liang J, Miao $Y$, Wang $M$, Yin W, et al. Clinical value of prophylactic radiotherapy after curative resection of esophageal carcinoma. Chinese Journal of Oncology, 2002, 24(6): 608-11.

19. Fok M, Sham JS, Choy D, Cheng SW, Wong J. Postoperative radiotherapy for carcinoma of the esophagus: a prospective, randomized controlled study. Surgery 1993;113:138-47.

20. Ténière P, Hay JM, Fingerhut A, Fagniez PL. Postoperative radiation therapy does not increase survival after curative resection for squamous cell carcinoma of the middle and lower esophagus as shown by a multicenter controlled trial. French University Association for Surgical Research. Surg Gynecol Obstet 1991;173:123-30.

21. Wu AJ, Bosch WR, Chang DT, Hong TS, Jabbour SK, Kleinberg LR, et al. Expert consensus contouring guidelines for intensity modulated radiation therapy in esophageal and gastroesophageal junction cancer. Int J Radiat Oncol Biol Phys 2015; 92: 911-20.

22. Wang X, Miao C, Chen Z, Li W, Yuan S, Yu J, et al. Can involved-field irradiation replace elective nodal irradiation in chemoradiotherapy for esophageal cancer? A systematic review and meta-analysis. Onco Targets Ther 2017: 10: 2087-95.

23. Cheng YJ, Jing SW, Zhu LL, Wang J, Wang L, Liu Q, et al. Comparison of elective nodal irradiation and involved-field irradiation in esophageal squamous cell carcinoma: a meta-analysis. Radiat Res. 2018; 59(5): 604-15.

24. Lu JC, Tao H, Zhang YQ, Zha WW, Qian PD, Li F, et al. Extent of prophylactic postoperative radiotherapy after radical surgery of thoracic esophageal squamous cell carcinoma. Dis Esophagus. 2008;21(6):502-507. 
25. Mei ZR, Xiang QC, Wu WJ, et al. Prospective study on preventive radiotherapy after esophageal cancer surgery[J]. Chin J Radiat Oncol, 1997;6(4):188-189.

26. Zieren HU, Müller JM, Jacobi CA, Pichlmaier H, Müller RP, Staar S. Adjuvant postoperative radiation therapy after curative resection of squamous cell carcinoma of the thoracic esophagus: a prospective randomized study. World J Surg. 1995;19(3):444-449.

27. Bédard EL, Inculet RI, Malthaner RA, Brecevic E, Vincent M, Dar R. The role of surgery and postoperative chemoradiation therapy in patients with lymph node positive esophageal carcinoma. Cancer. 2001;91(12):2423-30.

28. Cai WJ, Xin PL. Pattern of relapse in surgical treated patients with thoracic esophageal squamous cell carcinoma and its possible impact on target delineation for postoperative radiotherapy[J]. Radiother Oncol. 2010;96(1): 104-107.

29. Doki Y, Ishikawa O, Takachi K, Miyashiro I, Sasaki Y, Ohigashi H, et al. Association of the primary tumor location with the site of tumor recurrence after curative resection of thoracic esophageal carcinoma. World J Surg. 2005;29(6):700-707.

30. Lin SH, Wang L, Myles B, Thall PF, Hofstetter WL, Swisher SG, et al. Propensity score-based comparison of long-term outcomes with 3-dimensional conformal radiotherapy vs intensity-modulated radiotherapy for esophageal cancer[J]. Int J Radiat Oncol Biol Phys 2012; 84: 1078-85.

31. Dong Y, Guan H, Huang W, Zhang Z, Zhao D, Liu Y, et al. Precise delineation of clinical target volume for crossing-segments thoracic esophageal squamous cell carcinoma based on the pattern of lymph node metastases. J Thorac Dis. 2015;7(12):2313-2320

32. Liu J, Cai X, Liu Q, Li H, Cheng Y, Fu X. Characteristics of the local recurrence pattern after curative resection and values in target region delineation in postoperative radiotherapy for lower thoracic esophageal squamous cell cancer. Thorac Cancer. 2017;8(6):630-633.

33. Zhu Y, Li M, Kong L, Yu J. Postoperative radiation in esophageal squamous cell carcinoma and target volume delineation. Onco Targets Ther. 2016;9:4187-4196.

34. Shim YM, Kim HK, Kim K. Comparison of survival and recurrence pattern between two-field and three-field lymph node dissections for upper thoracic esophageal squamous cell carcinoma. J Thorac Oncol. 2010; 5(5):707-12.

35. Law S, Wong J. Two-field dissection is enough for esophageal cancer. Dis Esophagus. 2001;14(2):98-103. Doi: 10.1046/j.1442-2050.2001.00164.x

36. Chen J, Zhu J, Pan J, Zhu K, Zheng X, Chen M, et al. Postoperative radiotherapy improved survival of poor prognostic squamous cell carcinoma esophagus. Ann Thorac Surg. 2010;90(2):435-442.

37. Xiao ZF, Yang ZY, Liang J, Miao YJ, Wang LJ. Value of radiotherapy after radical surgery for esophageal carcinoma: a report of 495 patients. Ann Thorac Surg. 2003;75(2):331-336

38. Wang S, Wang Z, Yang Z, Liu Y, Liu X, Shang B, et al. Postoperative radiotherapy improves survival in stage pT2N0M0 esophageal squamous cell carcinoma with high risk of poor prognosis. Ann Surg Oncol. 2016;23(1):265272.

39. Prenzel KL, Bollschweiler E, Schroder W, Mönig SP, Drebber U, Vallboehmer D, et al. Prognostic relevance of skip metastases in esophageal cancer. Ann Thorac Surg. 2010;90(5): 1662-67.

40. Tachibana M, Dhar DK, Kinugasa S, Kotoh T, Shibakita M, Ohno S, et al. Esophageal cancer with distant lymph node metastasis: prognostic significance of metastatic lymph node ratio. J Clin Gastroenterol. 2000;31(4):318-22.

41. Fogliata A, Nicolini G, Clivio A, Vanetti E, Laksar S, Tozzi A, et al. A broad scope knowledge based model for optimization of VMAT in esophageal cancer: validation and assessment of plan quality among different treatment centers [J]. Radiat Oncol. 2015; 10:220.

42. Munch S, Aichmeier S, Hapfelmeier A, Duma MN, Oechsner M, Feith M, et al. Comparison of dosimetric parameters and toxicity in esophageal cancer patients undergoing 3D conformal radiotherapy or VMAT. Strahlenther Onkol. 2016; 192(10):722-29.

43. Shi L, Lai Y, Chen S, Fu L, Lin Q. Dosimetric superiority of IMRT with jaw tracking technique for whole esophagus and T-shaped field radiotherapyin advanced esophageal cancer. PLoS ONE 2018; 13(9):e0202628.

44. van Hagen P, Hulshof MC, van Lanschot JJ, Steyerberg EW, van Berge Henegouwen MI, Wijnhoven BP, et al. Preoperative chemoradiotherapy for esophageal or junctional cancer. N Engl J Med. 2012 May 31;366(22):2074-84.

45. Shim YM, Kim HK, Kim K. Comparison of survival and recurrence pattern between two-field and three-field lymph node dissections for upper thoracic esophageal squamous cell carcinoma. J Thorac Oncol. 2010; 5(5):707-12.

46. Sadrizadeh A, Bagheri R, Soltani E, Anvari K, Toussi MS, Moadikhah S. The Comparison of the Advantages of Neoadjuvant Chemoradiotherapy versus Postoperative Chemoradiotherapy: Outcomes in Esophageal Cancer Patients. J Gastrointest Cancer. 2018;49(1):50-56

47. Chen HS, Wu SC, Hsu PK, Huang CS, Liu CC, Wu YC. The prognostic impact of preoperative and postoperative chemoradiation in clinical stage II and III esophageal squamous cell carcinomas: a population based study in Taiwan. Medicine (Baltimore). 2015;94(25):e1002. 